\title{
Identification of immature granulocytes in cancer chemotherapy patients by cell counting vs. microscopic examination of blood smears
}

\author{
YI-QUN CHE $^{1}$, DI SHEN ${ }^{1}$, SHI-MIN ZHANG ${ }^{2}$ and JUN QI ${ }^{1}$ \\ ${ }^{1}$ Clinical Laboratory, Cancer Hospital, Chinese Academy of Medical Sciences and Peking Union Medical College; \\ ${ }^{2}$ Department of Clinical Laboratory, Peking Union Medical College Hospital, Beijing 100021, P.R. China
}

Received July 26, 2013; Accepted November 11, 2013

DOI: $10.3892 /$ mco.2014.243

\begin{abstract}
Tumor cell formation occurs through various mechanisms that may result in the growth of tumor blood vessels. Thus, novel methods are required to provide tumor therapy. The aim of the present study was to investigate the reliability of the abnormality-indicating alarm information provided by the automatic hematology analyzer in the measurement of immature granulocytes (IG) and assess the factors involved. The quality control groups at three concentration levels were repeatedly determined within 20 days to observe the within-run precision. The quality control groups at two concentration levels were repeatedly determined within 20 days to observe the between-run precision. The results obtained by the two methods were compared and the reliability of IG measurement using the hematology analyzer was analyzed. Additionally, IG parameters of 120 venous blood specimens collected from cancer patients were measured by cell counting using a hematology analyzer and stained blood smears were observed. The within-run precision experiment showed that the coefficient of variation $(\mathrm{CV})$ values of the IG percentage in the high, middle and low concentration levels were $3.62,6.75$ and $13.69 \%$, respectively, while the $\mathrm{CV}$ values of the IG percentage in the two-level between-run precision experiment were 5.6 and $7.1 \%$, respectively. All of the $\mathrm{CV}$ values were $<15 \%$ and within the permitted range. The true-positive rates obtained using the hematology analyzer in the $\mathrm{IG} \leq 1 \%, 1<\mathrm{IG} \leq 10 \%$ and $\mathrm{IG}>10 \%$ groups were $11.5,65.4$ and $95.0 \%$, respectively, and the false-negative rates were 0 . The IG percentages obtained through the hematology analyzer measurement and from the microscopic observation were $(6.98 \pm 11.18)$ and $(9.36 \pm 20.71) \%$, respectively. Results of
\end{abstract}

Correspondence to: Professor Jun Qi, Clinical Laboratory, Cancer Hospital, Chinese Academy of Medical Sciences and Peking Union Medical College, 17 Panjiayuan Nanli, Chaoyang, Beijing 100021, P.R. China

E-mail: qijun5610@126.com

Key words: immature granulocytes, cancer patients, cell counting, microscopic examination, smear the correlation analysis revelaed that there was an excellent correlation between the two methods $(r=0.1364)$. A significant difference between the two methods was observed using the signed-rank test $(\mathrm{P}=0.001)$. Manual microscopic observation is essential for the IG specimen that has been measured using an automatic hematology analyzer and has received an abnormality alarm. For cancer patients who receive chemotherapy, this application helps to provide laboratory data for clinical disease diagnosis and treatment monitoring.

\section{Introduction}

Previous studies focusing on mouse tumor models and cancer patients have revealed that tumor cells are able through various mechanisms to enhance the growth of myeloids and thus the development of leukocytosis, which is a sign of tumor formation, as well as inhibit the differentiation of myeloids and induce the natural change of these cells and the accumulation of immature myeloids $(1,2)$. This finding demonstrated the long-recognized correlation between tumors and leukocytosis. The neoplastic proliferation of myeloids is an indication of tumor formation; however, the increased immature myeloids may induce the development of the host's anti-tumor immune suppression and the growth of tumor blood vessels (3). Thus, tumor-related myeloid proliferation suggests a new treatment target is required in tumor therapy. The identification of immature granulocytes (IG) provides a more sensitive indicator for the tumor-related myeloid proliferation compared with white blood cell counting, the increase of granulocytes, the increase of monocytes or the decrease of lymphopenias and it realizes the early detection of tumor-related myeloid proliferation. Although not every cancer patient has an increased IG level, the IG parameter values in the diagnosis of tumors demonstrated that the most common routine blood examinations are fully utilized for the screening of tumor patients at the early stage or the preliminary and auxiliary discriminant stage of benign and malignant tumors.

The results obtained by the two methods were compared and the reliability of IG measurement using the hematology analyzer was assessed. Additionally, IG parameters of 120 venous blood specimens collected from cancer patients who received chemotherapy were measured by cell counting 
Table I. Within-run precision.

\begin{tabular}{lrrr}
\hline Variables & High level & Middle level & Low level \\
\hline WBC (G/l) & $20.60 \pm 0.16$ & $7.69 \pm 0.06$ & $1.89 \pm 0.03$ \\
RBC (T/l) & $5.69 \pm 0.02$ & $4.58 \pm 0.04$ & $2.29 \pm 0.01$ \\
HGB (g/l) & $185.70 \pm 0.82$ & $137.10 \pm 0.88$ & $59.10 \pm 0.32$ \\
HCT (l/l) & $55.13 \pm 0.25$ & $40.57 \pm 0.39$ & $17.82 \pm 0.11$ \\
MCV (fl) & $96.89 \pm 0.41$ & $88.65 \pm 0.23$ & $77.89 \pm 0.26$ \\
MCH (pg) & $32.64 \pm 0.13$ & $29.96 \pm 0.35$ & $25.85 \pm 0.16$ \\
MCHC (g/l) & $336.90 \pm 1.79$ & $338.00 \pm 4.06$ & $331.50 \pm 2.22$ \\
PLT (G/l) & $500.10 \pm 12.61$ & $251.30 \pm 7.79$ & $66.20 \pm 2.49$ \\
\hline
\end{tabular}

WBC, white blood cell; RBC, red blood cell; HGB, hemoglobin; HCT, hematocrit; MCV, mean cell volume; MCH, mean cell hemoglobin; MCHC, mean corpuscular hemoglobin concentration; PLT, platelets.

using a hematology analyzer and stained blood smears were observed under a microscope.

\section{Materials and methods}

Samples. Blood specimens were obtained for routine blood examination from chemotherapy patients presenting at the Cancer Hospital, Chinese Academy of Medical Sciences and Peking Union Medical College. Blood was collected (2 ml) in vials under vacuum, EDTA K3 was added to the vials and then they were gently agitated to prevent clotting. In total, 35 venous blood specimens from healthy subjects were used as the control group.

Measurement methods. The specimens were measured using the Celltac Es hematology analyzer (Nihon Kohden Corp, Tokyo, Japan) and the amounts of specimens to be measured were determined depending on the identified IG values. After the smears were prepared and stained using the Wright-Giemsa dye, they were observed under a microscope. The specimens were treated within $4 \mathrm{~h}$. Two treated smears were provided to two qualified laboratory physicians to classify the cells manually, in which 200 cells were observed under a high-power microscope and the percentage of white blood cells in different development stages was recorded. The classification procedure was single-blinded, and the average amount of cells in each different development stage between the two physicians were considered as the final results. Positive cut-off criteria by microscopy observation for the late promyelocytic stage or earlier were: blasts $>1 \%$, the variant $\mathrm{LY}>5 \%$ and nucleated red blood cells $(\mathrm{NRBC})>1 \%$ in white blood cell counting. If the observation showed that the primitive cells, early and middle promyelocytic cells, or late promyelocytic cells were $\geq 1 \%$, respectively, a positive result was noted.

At the same time, the IG percentage was determined in accordance with the server parameters provided by the hematology analyzer.

Scoring. If the result was negative in both the hematology analyzer measurement and the microscopic observation, it was considered 'true negative'; if the result was positive using the two methods, it was considered 'true positive'; if the
Table II. Positive results of microscopic observation corresponding to each group of immature granulocytes. ${ }^{\text {a }}$

\begin{tabular}{lc}
\hline Group & Positive \% in smear $(\mathrm{n} ; \%)$ \\
\hline A $(\mathrm{n}=26)$ & $3(11.5)$ \\
B $(\mathrm{n}=78)$ & $51(65.4)$ \\
C $(\mathrm{n}=20)$ & $19(95.0)$ \\
\hline
\end{tabular}

${ }^{a}$ Results obtained from the hematology analyzer.

result obtained from the hematology analyzer measurement was negative but positive in the microscopic observation, it was 'false negative'. The true-positive rate was calculated using the formula: 'true positive'/('true positive' + 'false negative') x $100 \%$.

Statistical analysis. The correlation of results obtained from the hematology analyzer and the microscopic observation were analyzed using the Spearman test and any difference between the two methods was observed using the signed-rank test.

\section{Results}

The quality control groups at the three concentration levels were repeatedly determined within 20 days to observe the within-run precision (Table I). The detection sensitivity of the automated instrumentation was $95 \%$, the specificity was $78 \%$, the positive predictive value was $76.1 \%$ and the negative predictive value was $99 \%$. For the microscopic observation method, IG as a percentage was calculated as: early promyelocytic rate + middle promyelocytic rate + late promyelocytic rate. The total counting results of each channel were presented as the server parameters of IMI TOTAL and DIFF TOTAL. Thus, the ratio of the two results may reflect the IG percentage. The true-positive rate was identified as $76.1 \%(67 / 88)$ in solid tumor, that of leukemia was $87.5 \%$ (28/32), while the false-positive rate in inpatients as compared to outpatients in solid tumor was 14.7 vs. $9.1 \%$. The majority of IGs in the IMI scatter plot coincided with microscopic examination of 
A

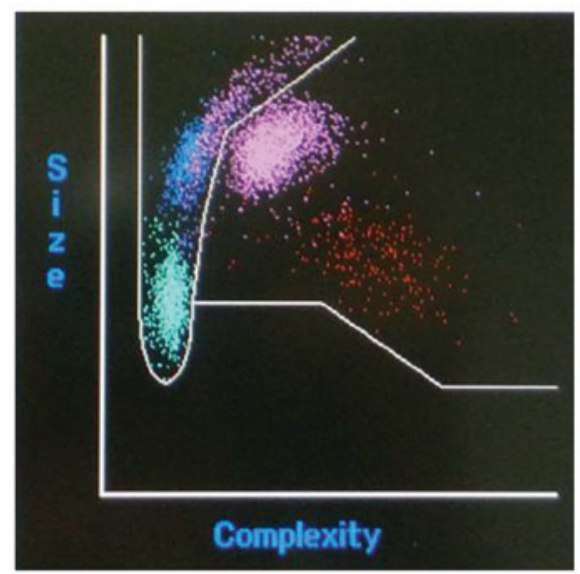

C

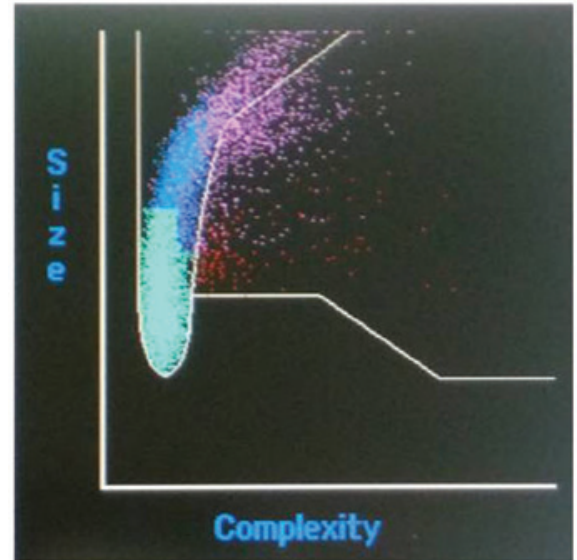

$\mathbf{E}$

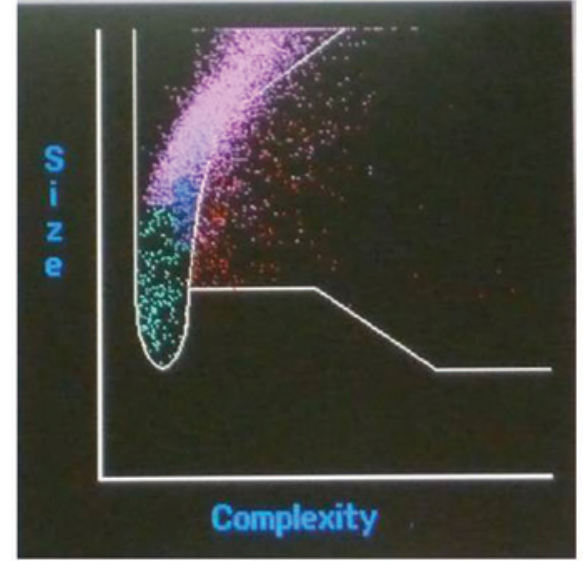

B

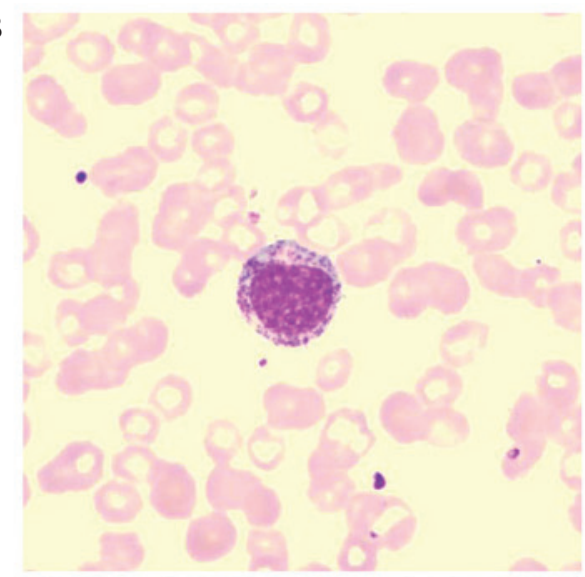

D

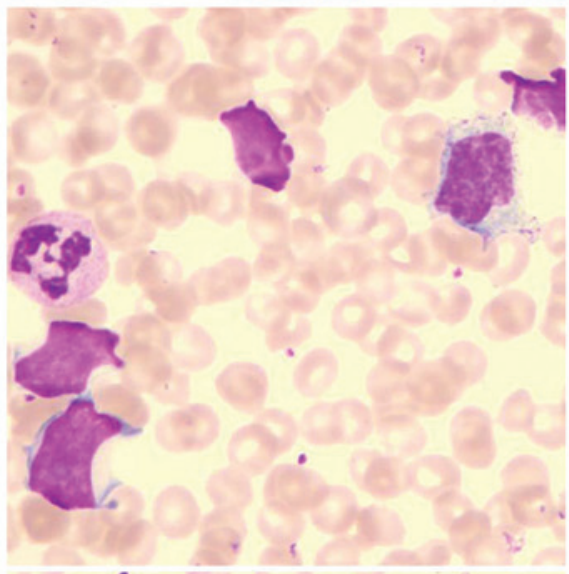

$\mathbf{F}$

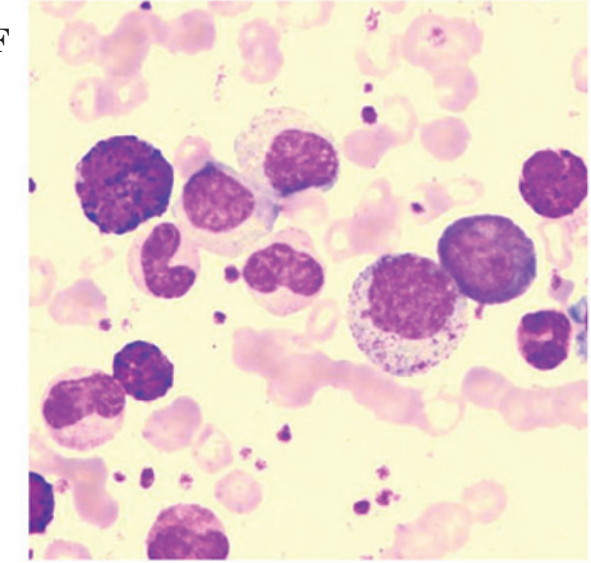

Figure 1. WBC counting and classification. The different immature granulocytes occupied different positions in the IMI scatter plot vs. microscopic examination of blood smears. (A) IG count $8.3 \%$, (B) myeloblasts $2 \%$, middle promyelocytic cells $2 \%$ and late promyelocytic cells $1 \%$. (C) IG count $9.7 \%$, (D) atypical lymphocytes $45 \%$ and promyelocytic cells $7 \%$. (C and D) The experiment showed that the instrument was inadequate to identify the myeloblasts and atypical lymphocytes. (E and F) IG parameter of chronic myelogenous leukemia cells was closely related to the positive rate in the microscopic examinations.

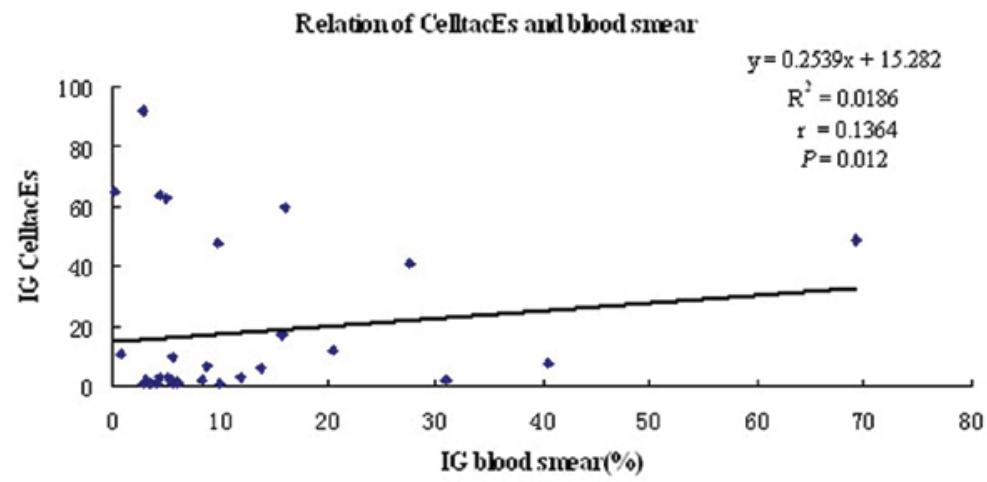

Figure 2. Immature granulocytes (IG) result in a correlation between the hematology analyzer measurement and the microscopic observation. 
blood smears (Fig. 1A and B). The results showed that the instrument was inadequate to identify the myeloblasts and atypical lymphocytes (Fig. 1C and D), while the IG parameter of chronic myelogenous leukemia cells was closely associated with the positive rate in the microscopic examinations (Fig. 1E and F).

The IG percentage was $(6.981 \pm 11.1853)$ vs. $(9.36 \pm 20.710) \%$ in the hematology analyzer measurement and the microscopic observation, respectively. The correlation analysis revealed that there was an excellent correlation between the two methods with $\mathrm{r}=0.1364$ and $\mathrm{P}=0.012$ (Fig. 2). A significant difference between the two methods was observed using the signed-rank test $(\mathrm{z}=-3.236, \mathrm{P}=0.001)$ and the results obtained from the hematology analyzer were higher than those obtained from the microscopic observation.

The IG results obtained from the hematology analyzer of 94 specimens were divided into 3 groups: Group A, IG $\leq 1.00 \%$; group $\mathrm{B}, 1.00 \%<\mathrm{IG} \leq 10.00 \%$; group $\mathrm{C}, \mathrm{IG}>10.00 \%$. In the microscopic observation, $\mathrm{IG}>0.5 \%$ was considered a positive result. The results showed that a false-positive result was obtained in the hematology analyzer measurement (Table II). There was an increase of neutrophils in $54.90 \%$ of false-positive specimens, atypical lymphocytes in $23.59 \%$, monocytes in $11.77 \%$, and eosinophils and basophils in $9.80 \%$, respectively.

\section{Discussion}

The performance verification and quality control of the IG parameters were carried out by comparing the results obtained from the microscopic observation and the automated instrumentation. Previously, it was shown that the most common causes for individuals $>10$ years were hematologic malignancies, chemotherapy and severe infections (4). The detection sensitivity of the automated instrumentation was $95 \%$, the specificity was $78 \%$, the positive predictive value was $76.1 \%$ and the negative predictive value was $99 \%$. The number of IG and the immature myeloid information were significantly elevated in neonates with early-onset sepsis compared to controls. IG and immature myeloid information are considered useful adjunctive methods in the diagnosis of neonatal early-onset sepsis (5). IG, which may be used for screening for bacteremia, with higher values of IGC $>0.3$ amd IG $(\%)>3$ exhibiting a specificity of $>90 \%$ (6). Thus, an IG parameter at its normal level is a reliable indicator for excluding abnormal myeloids. However, for a false-positive IG parameter, the microscopic observation is required to exclude or confirm the result.

Results of the present study have demonstrated that the percentage required to obtain a false-positive result in NRBC was relatively increased, and it may have been caused by the influence of cell debris, platelet aggregation and small lymphocytes. The coincidence rate for the alarm of blasts between the automated instrumentation method and the manual microscopic observation method was as high as $62 \%$, which specifically reflects the existence of blasts in the peripheral blood of patients. Thirty-five specimens, not alarmed in the automated instrumentation, were re-examined by microscopy and none of these specimens were reported to be positive. Of the 21 patients whose blood specimens were alarmed with blasts and reported to be positive in the microscopic examination, 10 cases were undergoing chemotherapy treatment and their illnesses were being partly or completely relieved with $\mathrm{WBC}<1.0 \times 10^{9} / 1$. In this case, the microscopic examination was prone to miss the positive result, however, the automated measurement was able to examine $>10,000$ cells. Thus, the hematology analyzer is more sensitive than the manual detection in the identification of blasts.

Of the 49 patients whose blood specimen examination yielded an IG alarm, 18 cases were leukemia patients who were administered with G-CSF subsequent to chemotherapy treatment. These patients were suffering from myelofibrosis with a WBC level $>10.0 \times 10^{9} / 1$ and a neutrophil level $>80 \%$. Toxic changes and degenerative changes were observed in the granulocytes of these patients, which may be a significant reason for the false-positive results in the automated measurement. Blood counts and peripheral smear findings may provide insight into the likelihood of a clonal etiology (7).

In general, if no alarm is utilized in the examination using a CelltacES hematology analyzer and there is no special clinical requirement, there is no need for manual detection. If there is an abnormal alarm or the clinical physician requires one, a manual microscopic examination is a requirement. The reason for a higher false-positive rate in inpatients over outpatients may be explained by the fact that the drugs affect the morphology and structure of the leukocytes yielding incorrect results by the instrument. For cancer patients undergoing radiotherapy or chemotherapy, the false alarm is more significant in their routine blood examination. The alarm signal administered to the leukemia patients was identical to the manual microscopic observation results, which proved that the CelltacEs has a relative higher reliability in the screening of leukemia. However, in the case of the negative results of the IG alarm, the possibility of IG should not be excluded. The experiment showed that the instrument was inadequate to identify the myeloblasts in Fig. 1D, while the IG parameter of chronic myelogenous leukemia cells was closely connected with the positive rate in the microscopic examinations (Fig. 1F).

Quantitative IG analysis of peripheral blood is one of the directions in the development of the blood cell analyzer, and it is an effective solution in overcoming the time-consuming and laborious shortcomings of smear microscopy. IG measurement has been suggested as a more readily available indicator of the presence of granulocyte precursors (left shift) (8). However, due to the constant alterations in the morphology of blood cells, the hematology analyzer cannot replace the morphological observation of the smears. It is recommended by the International Society for Laboratory Hematology (ISLH). The ISLH has been instrumental in standards and guidelines development in laboratory hematology and has also expanded its repertoire of disciplines to cater for the growing needs of laboratory hematologists by an international group. ISLH has stipulated that the advantages of both the microscopic examination and instrument measurement should be combined and the number of smears for microscopic observation should be reduced as much as possible based on the premise of ensuring the quality of results of the instrumental analysis. The IG percentage is primarily known through instrument analysis and a targeted smear observation helps to improve the reliability of the results obtained in the blood assay.

In conclusion, the information provided by the original instrument detection parameters can be used to determine the 
IG percentage primarily and provide a basis for additional examination. If there is a false-positive result, the microscopic examination should not be omitted. For clinically suspicious specimens particularly those of $\mathrm{IG} \geq 1 \%$, the microscopic examination should be actively performed to prevent misdiagnosis.

\section{Acknowledgements}

Supported by a grant from the National Natural Science Foundation of China (81000977).

\section{References}

1. Mantovani A, Allavena P, Sica A and Balkwill F: Cancer-related inflammation. Nature 454: 436-444, 2008.

2. Nozawa $\mathrm{H}$, Chiu $\mathrm{C}$ and Hanahan D: Infiltrating neutrophils mediate the initial angiogenic switch in a mouse model of multistage carcinogenesis. Proc Natl Acad Sci USA 103: 12493-12498, 2006
3. Wilcox RA: Cancer-associated myeloproliferation: old association, new therapeutic target. Mayo Clin Proc 85: 656-663, 2010.

4. Roehrl MH, Lantz D, Sylvester C and Wang JY: Age-dependent reference ranges for automated assessment of immature granulocytes and clinical significance in an outpatient setting. Arch Pathol Lab Med 135: 471-477, 2011.

5. Cimenti C, Erwa W, Herkner KR, Kasper DC, Müller W and Resch B: The predictive value of immature granulocyte count and immature myeloid information in the diagnosis of neonatal sepsis. Clin Chem Lab Med 50: 1429-1432, 2012.

6. Senthilnayagam B, Kumar T, Sukumaran J, M J and Rao KR: Automated measurement of immature granulocytes: performance characteristics and utility in routine clinical practice. Pathol Res Int 2012: 483670, 2012.

7. Devitt KA, Lunde JH and Lewis MR: New onset pancytopenia in adults: a review of underlying pathologies and their associated clinical and laboratory findings. Leuk Lymphoma: Aug 20, 2013 (Epub ahead of print).

8. Bernstein LH and Rucinski J: Measurement of granulocyte maturation may improve the early diagnosis of the septic state. Clin Chem Lab Med 49: 2089-2095, 2011. 